\title{
Toxocara canis Mimicking a Metastatic Omental Mass from Sigmoid Colon Cancer: A Case Report
}

\author{
Han-Gil Kim ${ }^{1}$, Jung-Wook Yang ${ }^{2}$, Soon-Chan Hong ${ }^{1}$, Young-Joon Lee ${ }^{1}$, Young-Tae Ju ${ }^{1}$, Chi-Young Jeong ${ }^{1}$, \\ Jin-Kwon Lee ${ }^{3}$, Seung-Jin Kwag ${ }^{1}$ \\ Departments of ${ }^{1}$ General Surgery and ${ }^{2}$ Pathology, Gyeongsang National University Hospital, Gyeongsang National University School of \\ Medicine, Jinju; ${ }^{3}$ Department of General Surgery, Gyeongsang National University Changwon Hospital, Gyeongsang National University \\ School of Medicine, Changwon, Korea
}

Toxocara canis is an important roundworm of canids and a fearsome animal parasite of humans. Human infections can lead to syndromes called visceral larva migrans (VLM), ocular larva migrans, neurotoxocariasis, and covert toxocariasis. VLM is most commonly diagnosed in children younger than 8 years of age, but adult cases are relatively frequent among those infected by ingesting the raw tissue of paratenic hosts in East Asia. This research reports the case of a 59-year-old man with sigmoid colon cancer, who visited our institution for surgery. An intraperitoneal mass was found on preoperative computed tomography, and it was thought to be a metastatic mass from sigmoid colon cancer. A postoperative histologic examination and serum test showed eosinophilic granuloma due to toxocariasis. Diagnosis of VLM is often difficult and highly suspicious in adults. Researchers suggest, although rarely, that VLM be included in the differential diagnosis as a cause of intraperitoneal tumors.

Keywords: Colonic neoplasms; Neoplasm metastasis; Toxocara canis; Visceral larva migrans

\section{INTRODUCTION}

Toxocara canis is one of the dog's most common parasites and continues to stimulate public health concerns with high scientific interest [1]. Eggs in dog feces are spread within weeks after having been deposited in local environments [2]. Humans can also be infected with second stage larva of T. canis. The incidence of T. canis in occupationally exposed adults, such as dog breeders and veterinary staff, is higher than it is in the normal population [3]. Children can become infected after having accidentally ingested infected Toxocara eggs from larvae in soil or on other contaminated

Received: November 10, 2017 • Accepted: December 20, 2017

Correspondence to: Seung-Jin Kwag, M.D.

Department of General Surgery, Gyeongsang National University Hospital, Gyeongsang National University School of Medicine, 79 Gangnam-ro, Jinju 52727, Korea

Tel: +82-55-750-8091, Fax: +82-55-755-0274

E-mail:drksj77@naver.com

ORCID code: https://orcid.org/0000-0002-9267-9158

(c) 2018 The Korean Society of Coloproctology

This is an open-access article distributed under the terms of the Creative Commons Attribution NonCommercial License (http://creativecommons.org/licenses/by-nc/4.0) which permits unrestricted noncommercial use, distribution, and reproduction in any medium, provided the original work is properly cited. surfaces [4]. Having a puppy at home is a risk factor for toxocariasis [5]. In adult cases, uncooked pigs, lambs, cows, and chicken liver are sources of human T. canis infection, as is the consumption of products produced in marine mammals [6]. Human infections can lead to syndromes called visceral larva migrans (VLM), ocular larva migrans (OLM), neurotoxocariasis (NT), and covert toxocariasis (CT) [7-9]. The most common of these is VLM, a clinical manifestation of systemic organ involvement by Toxocara species. Classical presentations of VLM include fever, hepatomegaly, abdominal pain, pulmonary manifestations, and headache. The main laboratory test results are leukocytosis due to eosinophilia and elevated Immunoglobulin E levels [10]. VLM with involvement of the intra-abdomen, but not solid organs, is a rare finding. This research paper reports one case of human toxocariasis presenting with an intra-abdominal mass, which was suspected as being a metastatic mass from colon cancer.

\section{CASE REPORT}

This case report was approved by the Institutional Review Board of Gyeongsang National University Hospital. A 59-year-old man, who was a member of the crew on a seagoing ship, was admitted 
Volume 34, Number 3, 2018

for surgery on a sigmoid colonic lateral spreading tumor (LST). He had no previous medical history, except that he had been diagnosed with hypertension at a local hospital 10 years earlier and was on medication for that condition. Endoscopy was performed, a polypectomy and an endoscopic mucosal resection were used to remove multiple colonic polyps, and chronic nonspecific colitis or tubular adenoma was found. A LST of $4 \mathrm{~cm}$ in size was found 25 $\mathrm{cm}$ above the anal verge, so an endoscopic resection was impossible. Only a biopsy and clipping were performed on the distal margin. The endoscopic biopsy revealed a tubulovillous adenoma. Malignancy was not ruled out, so the researchers decided to perform a radical colectomy, as well as additional tests.

Initial laboratory studies showed a slight increase in the eosinophil ratio, but the other findings were normal. The patient's white bleed cell count was $8,620 / \mu \mathrm{L}$ and showed $8.7 \%$ eosinophil granulocytes. His other blood tests were normal. The patient tested negative for hepatitis B surface antigen and anti-hepatitis $C$ virus antibody and positive for anti-hepatitis B surface antibody. The circulating levels of carcinoembryonic antigen were checked at $0.93 \mathrm{ng} / \mathrm{mL}$ and were within normal limits. Computed tomography of the abdomen showed a polypoid mass, suspected as T3, in the sigmoid colon and an omental mass suspected of being a metastatic mass from colorectal cancer (Fig. 1). Positron emission tomography - computed tomography was not performed because the insurance did not cover this and cancer had not been confirmed. The patient was taken to the operating room, and a laparoscopic-assisted anterior resection was performed. An ulcerofungating mass with a size of $5 \mathrm{~cm} \times 4 \mathrm{~cm}$ was found in the sigmoid colon $25 \mathrm{~cm}$ above the anal verge. A mass of $2 \mathrm{~cm}$ in size was observed in the omentum near the distal transverse colon and was resected. This resection included the surrounding omentum. Frozen biopsy was performed on the mass, and the result showed that this mass was not malignant. On the 4th day after surgery, the complete blood count was checked. The white bleed cell count was $7,940 / \mu \mathrm{L}$, and eosinophils had increased to $26.4 \%$. On the 5 th and the 7 th days after surgery, eosinophils of $35.1 \%$ and $34.3 \%$ were reached, respectively. On the 6th day after surgery, a pathologic report was obtained. The sigmoid colon neoplasm was diagnosed as an adenocarcinoma for which the depth of invasion was limited to the submucosa, and the omental mass was diagnosed as eosinophilic granuloma, in which a degenerated foreign body was thought to be a dead parasite (Fig. 2). Based on the results, consultation on infectious diseases was carried out,

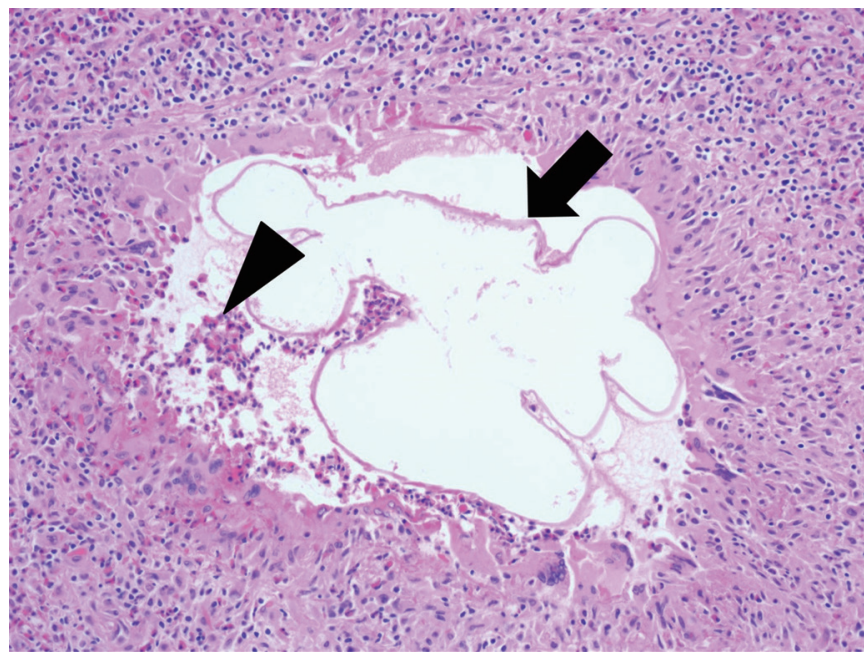

Fig. 2. Microscopic finding. Chronic granulomatous inflammation with a degenerated foreign body (arrow) and a moderate amount of eosinophilic infiltration (arrowhead).
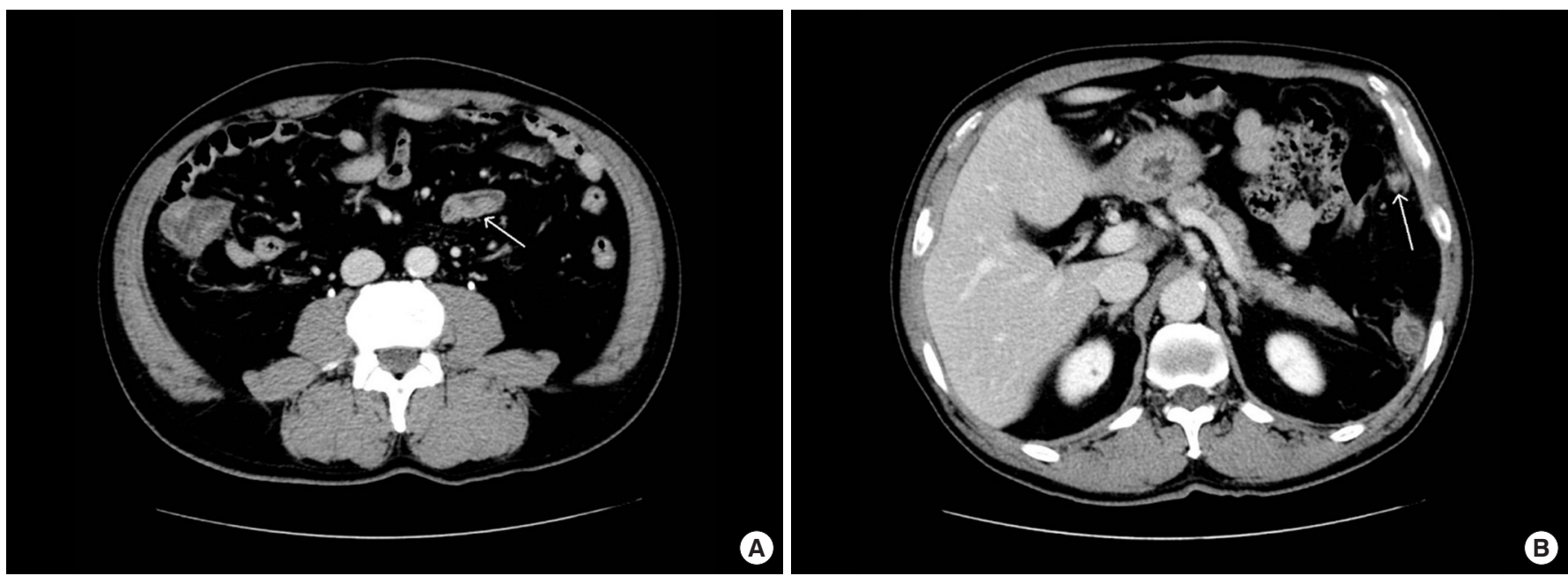

Fig. 1. (A) Contrast-enhanced abdominal computed tomography scan shows sigmoid colon cancer (arrow). (B) Contrast-enhanced abdominal computed tomography scan shows a left upper quadrant omental mass, suggestive of a metastatic mass from colon cancer (arrow). 
and albendazole was prescribed. An immunoassay was performed on the 7th day after the operation, and the patient was discharged. On the 12th day after the operation, test results were obtained. The Immunoglobulin G antibodies of T. canis were found to be positive, 1.414 based on a cut off 1.341. On the 15th day after the operation, the patient's visit to the outpatient clinic showed that eosinophils had decreased to $6.0 \%$.

\section{DISCUSSION}

Parasites infect about 2 billion people worldwide with parasitic nematodes, causing more than one billion people to suffer tremendous pain and massive economic loss. T. canis is an important roundworm of canids and a fearsome animal parasite of humans [11]. In Korea, Toxocara-seropositive rates were 51.2\% in total, $45.9 \%$ in urban areas and $56.8 \%$ in rural areas [12]. Various animals and people can become infected with T. canis, and infection of the human body can cause syndromes such as VLM, OLM, NT, and CT [7-9].

Toxocariasis is not very often fatal, but the inflammatory response to migrating larvae is associated with increased leukocytosis, including generalized lymphadenopathy, endophthalmitis, granulomatous hepatitis, asthma, endomyocarditis, and high eosinophilia $(>30 \%)$ [4]. Toxocariasis is a self-limiting disease. Therefore, patients without symptoms do not need medication. However, when symptoms such as eosinophilia or organ infiltration occur, administering albendazole at a dose of $800 \mathrm{mg}$ daily for 5 to 7 days is usually advisable [13]. If not treated in time, infection can lead to asthma and cause idiopathic seizure disorders or functional intestinal disorders. It can also cause skin disorders such as prurigo and urticaria $[4,5]$.

VLM is most commonly diagnosed in children younger than 8 years of age and is associated with recurrent larva infection or extreme discomfort caused by $T$. canis that can continue for weeks or months [9]. Adult cases are relatively frequent among those infected by ingesting the raw tissue of paratenic hosts in East Asia [14]. The patient in this case was a member of the crew on a seagoing ship and lived in an environment where eating raw food was prevalent, so infection with T. canis was possible. VLM syndrome usually includes fever, respiratory symptoms such as cough, dyspnea and bronchospasm, hepatomegaly, abdominal pain, and reduced appetite. Serology of Toxocara infection usually gives positive results [7]. The liver is the most commonly affected visceral organ in VLM. On computed tomography, liver lesions are not typically defined, and attenuated nodules are sometimes confused with metastatic cancers. Eosinophilic cholecystitis, large bowel masses, and ascites are rarely observed [8].

Georgiou et al. [5] reported on an ascending colon mass caused by VLM of T. canis. Park et al. [15] also reported cases where toxocariasis masqueraded as a liver or lung metastatic nodule in patients with gastrointestinal cancer. Lee et al. [12] reported that liver and lung nodules were more likely to be present as the grade of Toxocara increased. However, no cases of eosinophilic granuloma due to infections with T. canis were reported in the omentum. This is the case with patients who are not initially suspected of having Toxocara or other parasitic infections. In our case, preoperative computed tomography indicated that the mass was an intra-abdominal metastatic lesion of colon cancer. The researchers found a causative organism with serology for Toxocara after the pathologists showed the presence of nematodes for VLM. At the same time, the researchers found a rise in the eosinophil count in the complete blood count. Therefore, the features of this case suggested a diagnosis of parasite infestation in the intraperitoneal mass and the presence of parasites in the tissue, which may appear as metastatic lesions on preoperative imaging. A diagnosis of VLM is often difficult and is highly suspect in adults. Physicians should keep in mind a variety of symptoms, including eosinophilic granuloma, that mimic intraabdominal metastasis of colon cancer.

In conclusion, the researchers report the presence of an omental mass, a form of VML, which occurred due to T. canis infection. Such a mass is difficult to distinguish from intra-abdominal metastatic lesions of colorectal cancer. Eosinophilic granuloma caused by infection with a parasite such as T. canis, although rare, should be included in differential diagnoses as a possible cause of intraperitoneal tumors.

\section{CONFLICT OF INTEREST}

No potential conflict of interest relevant to this article was reported.

\section{REFERENCES}

1. Sudhakar NR, Samanta S, Sahu S, Raina OK, Gupta SC, Goswami TK, et al. Characterization of excretory-secretory antigens of adult Toxocara canis by western blotting. J Parasit Dis 2014;38: 166-9.

2. Shalaby HA, Abdel-Shafy S, Abdel-Rahman KA, Derbala AA. Comparative in vitro effect of artemether and albendazole on adult Toxocara canis. Parasitol Res 2009;105:967-76.

3. Deutz A, Fuchs K, Auer H, Kerbl U, Aspöck H, Köfer J. Toxocarainfestations in Austria: a study on the risk of infection of farmers, slaughterhouse staff, hunters and veterinarians. Parasitol Res 2005;97:390-4.

4. Despommier D. Toxocariasis: clinical aspects, epidemiology, medical ecology, and molecular aspects. Clin Microbiol Rev 2003; 16:265-72.

5. Georgiou C, Efstathiades Y, Dimitriou N, Theophanous M, Voros D. An unusual case of Toxocara canis of the ascending colon. Eur J Gastroenterol Hepatol. 2007;19:1149-53.

6. Goyette S, Cao Z, Libman M, Ndao M, Ward BJ. Seroprevalence of parasitic zoonoses and their relationship with social factors among the Canadian Inuit in Arctic regions. Diagn Microbiol In- 
fect Dis 2014;78:404-10.

7. Cooper PJ. Toxocara canis infection: an important and neglected environmental risk factor for asthma? Clin Exp Allergy 2008 Apr;38:551-3.

8. Rubinsky-Elefant G, Hirata CE, Yamamoto JH, Ferreira MU. Human toxocariasis: diagnosis, worldwide seroprevalences and clinical expression of the systemic and ocular forms. Ann Trop Med Parasitol 2010;104:3-23.

9. Macpherson CN. The epidemiology and public health importance of toxocariasis: a zoonosis of global importance. Int J Parasitol 2013;43:999-1008.

10. Arango CA. Visceral larva migrans and the hypereosinophilia syndrome. South Med J 1998;91:882-3.

11. Hotez PJ, Wilkins PP. Toxocariasis: America's most common neglected infection of poverty and a helminthiasis of global impor- tance? PLoS Negl Trop Dis 2009;3:e400.

12. Lee JY, Yang MH, Hwang JH, Kang M, Paeng JW, Yune S, et al. The prevalence of toxocariasis and diagnostic value of serologic tests in asymptomatic Korean adults. Allergy Asthma Immunol Res 2015;7:467-75.

13. Kim HB, Seo JW, Lee JH, Choi BS, Park SG. Evaluation of the prevalence and clinical impact of toxocariasis in patients with eosinophilia of unknown origin. Korean J Intern Med 2017;32:5239.

14. Akao N, Ohta N. Toxocariasis in Japan. Parasitol Int 2007;56:8793.

15. Park S, Kim YS, Kim YJ, Kyung SY, Park JW, Jeong SH, et al. Toxocariasis masquerading as liver and lung metastatic nodules in patents with gastrointestinal cancer: clinicopathologic study of five cases. Dig Dis Sci 2012;57:155-60. 